\title{
Stress echocardiography in the diagnosis of coronary artery disease: A systematic review
}

\author{
Jorge João dos S. Castro. Filho, ${ }^{* 1}$ Paulo M. Neufeld ${ }^{2}$
}

\begin{abstract}
Introduction: Coronary Artery Disease (CAD) contributes to the increase in the prevalence of cardiovascular disease, being the main cause of death worldwide. Objectives: This study compares the sensitivity of different methods of execution of Stress Echocardiography (SE) as to the accuracy in determining the presence of CAD, through a Systematic Literature Review (RSL). Methods: The databases of the Medical Literature Analysis and Retrieval System Online (MEDLINE), Latin American and Caribbean Literature in Health Sciences (Lilacs) and the Spanish Bibliographic Index of Health Sciences (IBECS) were searched through the Virtual Health Library (VHL) for articles published until December 2018 and including scientific articles published in journals, in any language, which met the following criteria: (A) Descriptive or analytical studies of primary or secondary basis and (B) Studies whose theme was the SE in adult coronary heart disease. Studies related to: (a) Elderly; (b) Animals; (c) Children and Adolescents; (d) Pregnant women; (e) Articles that alluded to left ventricular function, valvulopathies, aneurysms, endocarditis, arrhythmias, implanted electronic devices and other titles that were outside the scope of this work and (f) Articles published in languages other than English or Neolatin languages. Results: The study has selected 14 articles, which demonstrated that SE was accurate to diagnose CAD with sensitivities ranging from 41 to $100 \%$ with studies included within the normality curve, demonstrating that it is an excellent tool for the diagnosis of CAD compared to other methods.
\end{abstract}

Keywords: Coronary artery disease; Stress echocardiography; Systematic review.

\section{Introduction}

Coronary artery aisease (CAD) contributes significantly to the increased prevalence of cardiovascular disease, the leading cause of death worldwide. ${ }^{1}$

As for classification, CAD can be classified as chronic or acute, with treatment aimed at relieving anginal symptoms by preventing acute myocardial infarction (AMI) or premature death, consisting mainly of control and prevention of angina or reversal of plaque progression. ${ }^{2}$

In addition to clinical parameters, tests for the diagnosis of ischemia are disseminated by the guidelines of the American College of Cardiology. Noninvasivestress tests are appropriate in most patients with suspected CAD for diagnosis and risk stratification. ${ }^{3}$
1. Clínica de Cardiologia. Exército Brasileiro, Brazil.

2. Faculdade de Farmácia, Universidade Federal do Rio de Janeiro. Rio de Janeiro, RJ, Brazil.

* Correspondence address:

Clínica de Cardiologia, Exército Brasileiro

Endereço: Rua Moncorvo Filho, 34

Rio de Janeiro, RJ, Brasil

CEP: 20211-340.

E-mail: jjsfilho@hotmail.com

ORCID: https://orcid.org/ 0000-0002-6775-2392

BJHBS, Rio de Janeiro, 2021;20(1):27-35

DOI: $10.12957 /$ bjhbs.2021.59743

Received on 12/01/2020. Approved on 04/19/2021.

Coronary angiography (CAT) is considered the gold standard for the diagnosis of $\mathrm{CAD}$, although there are limitations and risks associated with procedure, ${ }^{4}$ after the initial electrocardiogram (ECG) test, patients who are able to exercise after the ECG, and have a low risk to intermediate CAD, they must be submitted to ECG Tests during exercise (Exercise Test). ${ }^{5}$

Those at high risk for CAD or who have an uninterpretable ECG character should be subjected to exercise stress using nuclear myocardial perfusion imaging tests or echocardiography, in patients who are unable to exercise, pharmacological stress is indicated in tests of myocardial perfusion or echocardiography. ${ }^{6}$

The Stress Echocardiography (SE) is a well established non-invasive technique that is most widely used in the evaluation of CAD. The specialization of professional requires proficiency in echocardiography transthoracic.? .

The obvious relevance of morbidity and mortality of $\mathrm{CHD}$ in $\mathrm{Brazil}^{8}$ and the world is constituted as a public health problem getting patent both the potential harm to patients and costs to the health system. Thus, this article aims to study the diagnostic sensitivity of $\mathrm{SE}$, according to the scientific literature, in order to contribute to public health, proposing to reaffirm the usefulness of this diagnostic tool for CAD. 


\section{Original article}

\section{Material and methods}

This study comprised a systematic review on SE, covering the scientific literature available until December 2018, according to the guidelines of the Preferred Reporting Items for Systematic Reviews and MetaAnalyzes Group Guidelines (PRISMA). ${ }^{9}$

For this systematic review, the following research question was elaborated: "Considering SE as a diagnostic tool, what is its sensitivity for the diagnosis of CAD and if there is a benefit in relation to other methods used for the same indication clinic?"

The Virtual Health Library (VHL) was defined to search the databases of the Medical Literature Analysis and Retrieval System Online ( MEDLINE), Latin American and Caribbean Literature in Health Sciences ( Lilacs ) through articles published until December 2018 .

As this is an exploratory systematic review, and because it is the first systematic review properly carried out on the topic in Brazil, it was decided to carry out a broader search, with high sensitivity. We obtained the terms Descriptors in Health Sciences ( DeCS) "Echocardiography under stress", which translated to the corresponding terms of the Medical Subject Headings ( MeSH ): "Echocardiography , Stress."

These descriptors were used to perform the first search via the VHL, in order to assess the accuracy of more sensitive searches - which included free search arguments - or more specific ones - which included the search arguments "Echocardiography ,Stress" and their versions or truncations.

As a result of this analysis: The following search expressions were used in the databases searched:

\section{VHL}

(tw:(stress echocardiography)) AND(tw:(coronary disease))

Scientific articles published in journals, in any language, that met the following criteria were included: (A) Descriptive or analytical studies of primary or secondary basis and (B) Studies whose subject is SE in adult CAD.

For articles published in languages other than Portuguese, English and Spanish, the Google Translate ${ }^{\mathrm{TM}}$ tool was adopted to support translation, to minimize publication bias by language.

Studies related to: (a) Elderly people; (b) Animals; (c) Children and Adolescents; (d) Pregnant women; (e) Articles that alluded to left ventricular function, valvulopathies, aneurysms, endocarditis, arrhythmias, implanted electronic devices and other titles that were outside the scope of this work and (f) Articles published in languages other than English or Neolatin languages.

The titles of all articles found in the search were reviewed, and those potentially linked to SE, were preserved. Abstracts were independently assessed by two reviewers, namely, the author and co-author , to determine whether such articles met the inclusion and exclusion criteria.

Assessment discrepancies between the two reviewers were resolved by consulting a third reviewer. If there was uncertainty as to whether a study met the inclusion criteria or not, the article was included in order to reduce the likelihood that a relevant study would be ignored.

During the complete revision of a text, the articles were again independently reviewed by the two reviewers and, if necessary, judged by the third reviewer, having performed the descriptive statistical analysis of the data using the SPSS software, version 20 for the average and standard deviation, and two normality tests, the Kolmogorov-Smirnov and Shapiro-Wilks with a $\mathrm{p}>=0.05$, that is, such studies meet normality , inserted from the data in Table 1.

The methodological quality of each article was assessed using the Quality Assessment Tool for Systematic Reviews of Observational Studies (QATSO) scale. ${ }^{10}$

During the process, QATSO can simplify and generalize the information that could be extracted, which is an inherent characteristic of the assessment tools. In this specific case, the total score is obtained divided by the total number of applicable items, and a study is considered bad from 0 to $33 \%$, satisfactory from 33 to $66 \%$ and good from 67 to $100 \%$.

After assessing the heterogeneity of the results, a choice was made between a model for presenting the results in a descriptive manner with statistical analysis. ${ }^{11}$ The presentation of the results and discussion of this study followed the Preferred Reporting Items for Systematic Reviews and MetaAnalyzes Group Guidelines (PRISMA) ${ }^{9}$

In the article selection phase, a total of 1108 potentially eligible publications on SE were found in the databases, published between 2013 and 2018,1054 in MEDLINE,39 in LILACS, 13 in IBECS and 2 in the State Health Secretariat of São Paulo State, making a total of 1108 titles.

Based on the selection, 43 were excluded by language and 457 by title, resulting in 608 articles that were selected for reading the abstracts, with 451 exclusions per abstract and a set of 157 articles selected for reading the full text, of which 14 were included in the review (Figure 1).

The quality methodology of the articles in agree- 
ment with the scale QATSO, ranged from good (two), satisfactory (eleven) and bad (one article).

A brief summary of the 14 articles included in this review is shown below, in Table 2, showing the context in which SE was assessed and its accuracy regarding the diagnosis of CAD

\section{Results and discussion}

In the recent scientific literature there are few studies on the sensitivity of SE for the diagnosis of CAD, however, with a cross-sectional design ${ }^{26}$ adequate to the proposed objectives, it was possible to carry out this review.
In general, when it has evaluated the diagnostic efficacy between SE and CAD the majority of authors contemporary to support this hypothesis is worth of studies based on research primary. However, it is possible to adopt another methodology that is equally efficient, such as systematic review. ${ }^{27}$

The findings of Alfaro et $\mathrm{a}^{12}$ demonstrated the authors' initial experience with the method using SE Dipiridamol , and despite not having verified the sensitivity of the procedure, they were able to verify the absence of significant adverse events.

The studies by Nagi et al., ${ }^{14}$ Park et al., ${ }^{19}$ Piccione et al., ${ }^{16}$ Aggeli et al., ${ }^{17}$ Ejlersen et al., ${ }^{20}$ Rumbinaite et al. ${ }^{21}$ and

Table 1. Characteristics of the studies regarding sensitivity

\begin{tabular}{|c|c|c|c|c|c|c|c|c|c|}
\hline Author & Sensitivity & ADA & Non-ADA & $\begin{array}{l}\text { Up to two } \\
\text { vessels }\end{array}$ & $\begin{array}{l}\text { Above two } \\
\text { vessels }\end{array}$ & $\begin{array}{l}\text { Change in } \\
\text { Contract \% }\end{array}$ & $\begin{array}{c}\text { Myocardial } \\
\text { strain analysis }\end{array}$ & Global & Maximum \\
\hline Alfaro et al. ${ }^{12}$ & NR & & & & & & & & \\
\hline Kamiya et al. ${ }^{13}$ & $50,00 \%$ & & & & & & & & \\
\hline Nagi et al. ${ }^{14}$ & & $86,00 \%$ & $85,00 \%$ & & & & & & \\
\hline Shehata. ${ }^{15}$ & & & & $100,00 \%$ & $90,30 \%$ & & & & \\
\hline Piccione et al. ${ }^{16}$ & $84,00 \%$ & & & & & $44,00 \%$ & $61,00 \%$ & $84,00 \%$ & \\
\hline Aggeli et al. ${ }^{17}$ & & & & & & $78,00 \%$ & & $81,00 \%$ & $91,00 \%$ \\
\hline Utsunomiya et al. ${ }^{18}$ & $80,00 \%$ & & & & & $52,00 \%$ & & & \\
\hline Park et al. ${ }^{19}$ & $48,00 \%$ & & & & & $91,00 \%$ & & $85,00 \%$ & \\
\hline Ejlersen et al. ${ }^{20}$ & & & & & & $69,00 \%$ & & & \\
\hline Rumbinaite et al. ${ }^{21}$ & $76,70 \%$ & & & & & & & $94,00 \%$ & \\
\hline Dattilo et al. ${ }^{24}$ & $94,60 \%$ & & & & & & & & \\
\hline Gurunathan et al. ${ }^{25}$ & $68,00 \%$ & & & & & & & & \\
\hline $\begin{array}{l}\text { AVERAGE } \\
\text { (Sensitivity) }\end{array}$ & $71,60 \%$ & $86,00 \%$ & $85,00 \%$ & $100,00 \%$ & $90,30 \%$ & $66,80 \%$ & $61,00 \%$ & $86,00 \%$ & $91,00 \%$ \\
\hline $\begin{array}{l}\text { STANDARD DEVIATION } \\
\text { (Sensitivity) }\end{array}$ & 17,39 & & & & & & & & \\
\hline Čelutkienè et $a .^{22}$ & $\begin{array}{c}91,6 \% / \\
76,8 \%\end{array}$ & & & & & & & & \\
\hline Kataoka et al. ${ }^{23}$ & $\begin{array}{c}46,0 \% / \\
22,3 \% / \\
55,3 \%\end{array}$ & & & & & & & & \\
\hline
\end{tabular}

Legend: NR = not reported; $A D A=$ Anterior Descending Artery.

Authorship: The authors (2020). 


\section{Original article}

Table 2. Results

\begin{tabular}{c|l|l}
\hline \multicolumn{1}{c}{ Identification } & \multicolumn{1}{c}{ Goals } & \multicolumn{1}{c}{ Results } \\
\hline 1 & $\begin{array}{l}\text { To demonstrate the experience with SE } \\
\text { Dipyridamole, as well as the study of } \\
\text { the Coronary Flow Reserve in the distal } \\
\text { portion of the Anterior Descending Artery } \\
\text { (ADA). }\end{array}$ & $\begin{array}{l}\text { A total of } 162 \text { patients selected completed the test in less than 25 } \\
\text { minutes without significant side effects. The test was positive for } \\
\text { ischemia only in 21\% of the patients. Due to the short follow-up } \\
\text { period and the low incidence of events, the predictive value cannot } \\
\text { be measured beyond sensitivity. }\end{array}$ \\
& &
\end{tabular}

2

To compare the diagnostic of potential functionally significant stenosis observed in RFF between Cardiac Magnetic Resonance with Myocardial Perfusion (RMC-MPI), Single Photon MPI Emission Computed Tomography (SPECT-MPI) and Dobutamine Stress Echocardiography (DSE).

To evaluate the viability of the analysis
of deformations based on tissue Strain
(ST) and tissue speed during the Stress
Echocardiogram with contrast, comparing
it with the SE using only the contrast
(WMS).

4

Evaluate the relationship between the ischemic threshold measured during the DSE and the CATE.

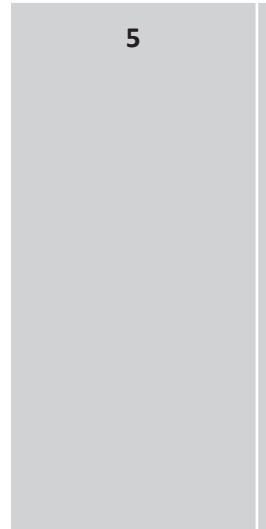

6
To investigate the additional value of SE sensitivity with assessment of the global myocardial ST compared to SE with assessment of wall contractility for the detection of CAD.

Determine the diagnostic value of the left ventricular (LV) longitudinal ST and torsion to diagnose CAD during DSE.
Patients with CAD in a cluster of 25 patients that had at least one angiographic $\geq 50 \%$ stenosis in coronary angiography. The sensitivity of RMC-MPI was significantly higher than that of SPECT-MPI and EED . RMC-MPI ob had the highest diagnostic sensitivity among all modalities tested in patients with CAD.

Echocardiograms with isolated contrast and in association with 60 patients were analyzed. At the patient level, the diagnostic accuracy of the combined method was not found to be superior to the WMS (82 versus $78 \%$ ); there was a tendency to improve the sensitivity and specificity to detect CAD in the right coronary artery circulation (sensitivity: 85 vs. $77 \%, P=N S$; specificity: 96 vs. $94 \%$ ).

Two hundred consecutive patients with positive EED test results were prospectively enrolled. The ischemic threshold was recorded for all patients using the formula defined by the American Society of Echocardiography. The ischemic threshold measured during high-dose DSE correlates significantly with the number of significantly stenosed coronary arteries, however, it does not correlate with the degree of vessel stenosis.

Fifty-two patients underwent DIP of dipyridamole followed by CATE within one week. The studied population was divided into two groups according to coronary angiographic findings: those with CAD and those without CAD. With regard to the results of the study, the accuracy in the detection of CAD, with assessment of the contractility of the wall, generated sensitivity of $44 \%$, specificity of $55 \%$, positive predictive value of $73 \%$ and negative predictive value of $26 \%$, with the Using the myocardial deformation analysis, the sensitivity was $61 \%$, the specificity was $90 \%$, the positive predictive value was $94 \%$ and the negative predictive value was $47 \%$. While the assessment of the global myocardial ST assessed as the difference between peak dose and resting values provided sensitivity of $84 \%$, specificity of $92 \%$, positive predictive value of $96 \%$ and negative predictive value of $68 \%$.

A sum of 100 patients with known or suspected coronary disease were studied, excluding those with a previous history of transmural infarction. The values related to the sensitivity and specificity for the difference in the score of the contractility of the wall were $78 \%$ and $88 \%$, respectively. The difference in the global longitudinal ST demonstrated a sensitivity of $81 \%$ and $72 \%$ of specificity for disease detection. The respective torsion values were $81 \%$ and $82 \%$. Combination of the difference in the contractility score of the wall with the torsion for detecting disease showed $91 \%$ sensitivity and $79 \%$ specificity. The authors concluded that the implementation of LV global and regional function marking tracking during DSE could serve as an adjunct method for assessing CAD, providing quantitative diagnostic information. 
Table 2. Results (continued)

\begin{tabular}{l|l|l}
\hline 7 & $\begin{array}{l}\text { To evaluate the diagnostic value of } \\
\text { echocardiographic findings at rest, } \\
\text { including the measurement of Total } \\
\text { Cardiac Calcification (CCT) in combination } \\
\text { with DSE for the detection of myocardial } \\
\text { ischemia. }\end{array}$ & $\begin{array}{l}\text { In total, } 110 \text { patients with suspected angina underwent resting } \\
\text { echocardiography and DSE. Based on resting echocardiography, } \\
\text { the CCT score, ADA diastole-systole velocity ratio and positive } \\
\text { myocardial velocity during fas and isovolumetric relaxation were } \\
\text { determined. They found that the DSE had excellent specificity } \\
(89 \%), \text { but modest sensitivity (52\%) for the analysis of the }\end{array}$ \\
$\begin{array}{ll}\text { Wall Contractility Abnormality (WMA). Multivariate analysis } \\
\text { showed that the CCT index } \geq 2 \text { and positive myocardial velocity } \\
\text { duration during the isovolumetric relaxation phase } \geq 71 \text { ms were }\end{array}$ \\
independent predictors of ischemia. The authors concluded that \\
the integration of DSE and resting echocardiographic findings that \\
describe the degree of cardiac calcification, impaired ADA flow and \\
the extension of the slow myocardial ejection phase improves the \\
detection of RFF-guided ischemia detected by CATE.
\end{tabular}

8

To investigate the role of specific analysis of the myocardial layer using twodimensional echocardiography with point tracking during stress infusion.
A total of 121 patients who underwent SE and presented normal wall contractility and ejection fraction during the initial examination were enrolled. All patients underwent CATE after SE in two weeks. Patients were divided into the following four groups according to the results of SE and the status of CAD: negative SE without significant CAD ( $n=73)$, positive SE with significant CAD $(n=16)$, negative SE with significant $\operatorname{CAD}(n=17)$ and positive $S E$ without significant CAD $(n=15)$. During echocardiography in the recovery phase, the assessment of the global longitudinal ST of the endocardium was significantly different between patients with and without CAD, regardless of the results of SE. However, the authors concluded that the diagnostic accuracy was improved by applying the results of the global longitudinal endocardial ST in compared to the visual estimation of $\mathrm{EE}$.

A total of 128 patients underwent SE, myocardial scintigraphy, exercise test and CAT. Forty-five patients had CAD. The sensitivity, specificity and accuracy of the methods were respectively for $\mathrm{EE}$, myocardial scintigraphy and exercise test: 69\%-84\%-79\%/82\%-94\%$90 \%$ and $73 \%-78 \%-77 \%$. Changes in global longitudinal endocardial ST and wall contractility during SE were closely associated with the presence of CAD and the combined sensitivity similar to that of myocardial scintigraphy. and exercise testing are the dominant methods of choice. The diagnostic value of the difference in the evaluation of the global longitudinal endocardial ST at rest and stress with the contractility of the wall using the SE with adenosine was compared to myocardial perfusion scintigraphy and exercise test with Duke score score, using the CAT as a reference.

Evaluate the following parameters: the assessment of global longitudinal ST, regional systolic and diastolic ST, deformation rate at rest and during DSE to detect significant coronary artery stenosis in patients with a moderate or high probability of CAD, DSE and MRI of adenosine.
The authors performed the assessment in 127 patients with a moderate and high probability of CAD and left ventricular ejection fraction $\geq 55 \%$. They showed that the assessment of global longitudinal ST is highly sensitive and specific for detecting hemodynamically significant coronary lesions, validated by secondary perfusion defects due to coronary artery stenosis in patients with moderate and high probability of CAD. 


\section{Original article}

Table 2. Results (continued)

\begin{tabular}{|c|c|c|}
\hline 11 & $\begin{array}{l}\text { To evaluate whether the combination } \\
\text { of several quantitative parameters in a } \\
\text { mathematical model would improve the } \\
\text { detection of myocardial ischemia during } \\
\text { DSE when compared to the conventional } \\
\text { analysis of the movement of the wall. }\end{array}$ & $\begin{array}{l}\text { A prospective study was carried out with } 151 \text { patients in the test } \\
\text { group and } 105 \text { patients in the validation group were selected } \\
\text { and submitted to SE between January } 2008 \text { and December } 2012 \text {. } \\
\text { Myocardial ischemia was detected visually in } 60 \text { ( } 39.7 \%) \text { and in } \\
58(54.2 \%) \text { patients in the test and validation group, respectively. } \\
\text { A total of } 76(50.3 \%) \text { patients in the test group and } 69 \text { patients } \\
(65.7 \%) \text { in the validation group had } \geq 50 \% \text { of coronary stenosis. } \\
\text { The sensitivity and specificity of the mathematical model per } \\
\text { patient in the test group were } 91.6 \% \text { and } 86.3 \% \text { compared to } \\
76.8 \% \text { and } 89.0 \% \text { of the visual assessment, respectively. The } \\
\text { authors concluded that the image of myocardial deformation can } \\
\text { potentially replace visual assessment with an automated prediction } \\
\text { model for detecting stress-induced ischemia. }\end{array}$ \\
\hline
\end{tabular}

12

To assess whether transient LV ischemic dilation can be considered an additional marker of myocardial ischemic response.

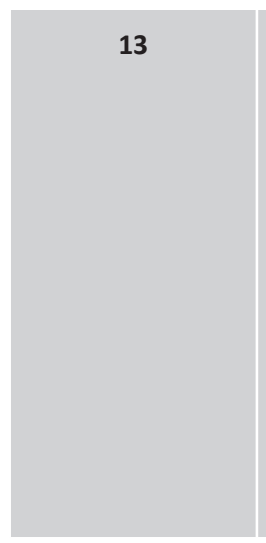

14

Evaluate patients with non-diagnostic results through SE with dipyridamole, verified by means of coronary angiotomography for the possible presence of CAD.

To determine the agreement between the wall thickening assessed during clinically indicated SE and RFF measurements and factors associated with adverse events in these patients.
A total of 88 patients underwent EED, where the final diastolic and stroke volumes were measured at rest and at peak stress stages, and the percentage of variation calculated. There were 27 (31\%) with transient ischemic dilation of the final systolic volume and $12(14 \%)$ of the final diastolic. For the detection of moderate or greater myocardial ischemia, the transient ischemic dilation of the final systolic volume showed a sensitivity of $46 \%$, specificity of $83 \%$, a positive predictive value of $70 \%$ and a negative predictive value of $64 \%$. The authors concluded that transient ischemic dilation during $\mathrm{SE}$ is a marker of extensive myocardial ischemia and can be used as an additional marker of greater risk.

Sixty-five consecutive patients who underwent undiagnosed SE were studied by two-dimensional echocardiography and coronary angiotomography. The assessment of global longitudinal and circumferential ST at rest and after stress were compared according to data derived from coronary angiotomography. The assessment of circumferential global ST at rest and after stress was similar in patients in the groups with positive and negative angiotomography. The assessment of global longitudinal ST at rest was significantly lower in patients with positive coronary angiotomography compared to the group with negative coronary angiotomography. The authors concluded, therefore, that the evaluation of ST during two-dimensional stress echocardiography allows, in non-diagnostic cases, to identify patients with moderate CAD with high sensitivity and specificity.

Two hundred and twenty-three consecutive patients undergoing measurements of SE and invasive RFF were retrospectively analyzed for diagnostic agreement and clinical outcomes. At the vessel level, sensitivity, specificity, positive predictive value and negative predictive value of SE to identify significant disease as assessed by the RFF was $68 \%, 75 \%, 43 \%$ and $89 \%$, respectively. There was a significant increase in the rate of events in patients with Wall Thickening Abnormalities (PRCA)/negative RFF versus without negative PRCA/RFF ( $P=0.01$ ), but not significant in positive PRCA / RFF. The authors concluded that in a population of patients with significant cardiovascular risk factors, a normal SE had a high negative predictive value to exclude abnormal RFF .

Authorship: The authors (2020). 
Dattilo et al. ${ }^{24}$ evaluated the sensitivity of SE associated with the use of tissue ST evaluation which conferred an increase in the sensitivity of the studies, reaching the same sensitivity to Myocardial Scintigraphy evaluated by the study by Ejlersen et al. ${ }^{20}$

In the study by Kamiya et al. ${ }^{13}$ the sensitivity was compared to diagnose functionally significant stenosis observed in RFF, between the RMC-MPI,SPECT-MPI and EED, with the RMC-MPI having the highest sensitivity, because the device used had high temporal resolution, making it possible to diagnose coronary disease earlier than changes in wall contractility identified with DSE.

Shehata ${ }^{15}$ demonstrated that the ischemic threshold measured during high-dose DSE correlates significantly with the number of significantly stenosed coronary arteries, making the method well used for the diagnosis of multivascular coronary lesion.

Utsunomiya et al. ${ }^{18}$ concluded that the integration of DSE and echocardiographic findings at rest that describe the degree of cardiac calcification, impaired ADA flow and the extension of the slow myocardial ejection phase improves the detection of RFF-guided ischemia detected by CATE.

Čelutkienè et al., ${ }^{22}$ Kataoka et al., ${ }^{23}$ and Gurunathan et al. ${ }^{25}$ concluded that the myocardial deformation assessed by SE is an important ischemia marker that in the case of Čelutkienè et al. ${ }^{22}$ could not be replaced by an automated model.

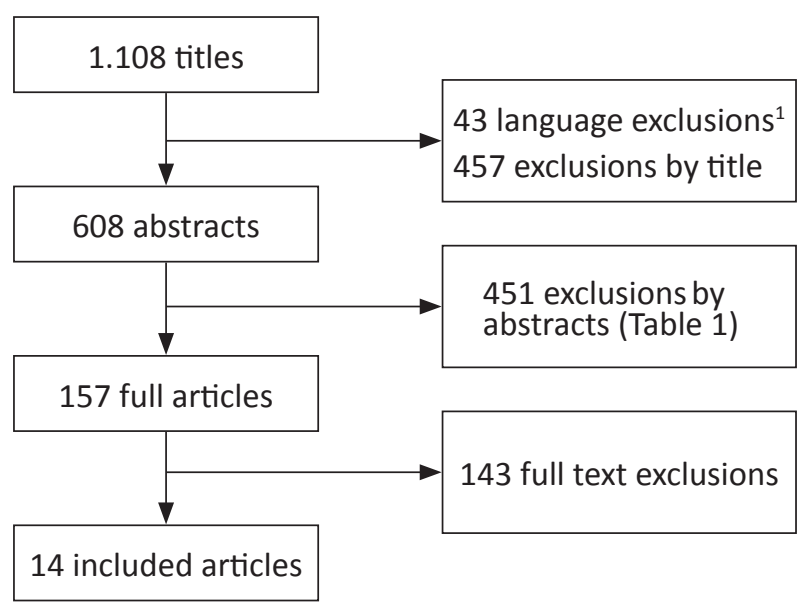

Figure 1. Selection process of the articles included in the review

${ }^{1}$ The languages of the articles excluded by this criterion were: German (12), Chinese (5), Hungarian (1), Japanese (1), Polish (5), Russian (14), Swedish (1), Turkish (2) and Ukranian (2).

Authorship: The authors (2020).
There was no considerable publication bias in the systematic review, caused by the exclusion of 43 articles published in languages other than English or Neolatin languages, as they could not be related to the research topic, this being an exploratory review, whose results produced a survey only descriptive statistics of SE sensitivity.

As a rule, the publication bias linked to the language has effects on the results of the systematic review in cases where studies of analytical epidemiology (clinical trials or analytical studies) are included, which did not occur in the present study. ${ }^{28}$

None of the articles included in this review adopted analytical designs that allowed the identification of confounding factors to obtain the sensitivity of the exam, therefore, there is a need to expand the scope of evidence available on the topic, which still requires original studies on the model.

The low rate of methodological information is a fact identified in the articles, which is evidenced by the low proportion of explicit description of the exclusion criteria, although data on the inclusion criteria are more frequently presented.

It is noted that the scientific evidence presented in general good methodological quality, since, according to the criteria adopted in this systematic review, only one article in 14 presented low or poor methodological quality.

We chose not to exclude the articles based on criteria of methodological quality, since this is the first systematic review on the subject, therefore presenting exploratory characteristics.

The fact that there are exams participating in the exclusion criteria (such as exams of children and adolescents) in some of the articles produces information bias in a small portion of the results of this study. However, the alternative would be to completely exclude articles with large samples and valid data.

It was considered that this exclusion would decrease the sensitivity of this systematic review, which would not be desirable, due to its exploratory nature, and due to the fact that it has a small number of articles, given the recent nature of the research on the topic.

There is an important factor to be mentioned that makes it difficult to expand the offer of the method, as the laboratory must have, in addition to the stressor, equipment such as electrocardiograph, defibrillator and a qualified team to provide advanced life support with the availability of all medications foreseen in the guidelines for this purpose. ${ }^{29}$ 


\section{Original article}

\section{Conclusion}

In patients with suspected CAD who are unable to perform an exercise test, with intermediate pretest probability, non-invasive diagnostic modalities available include Myocardial Scintigraphy, CMR and SE with dipyridamole, adenosine or dobutamine.

We have seen that in daily clinical practice there is no hard-and-fast rule that directs the performance of one or the other protocol for SE, as several factors influence this option.

Among the factors mentioned above, the most important are the non-existence of contraindications or conditions that reduce the safety of their performance and the professional's experience in relation to the modality, considering that the echocardiographic evaluation is still subjective and depends on training and training the learning curve of those who perform it.

It appears that there is no closed flowchart for choosing the method when it is intended to make a diagnosis and establish a prognosis in coronary disease, although EE, with its various modalities, has already established itself as a diagnostic alternative for several special groups of patients, with equivalent responses or even sometimes higher than those obtained by other complementary non-invasive diagnostic methods.

All methodologies have applications, limitations

\section{Referências}

1. Neumann FJ, Sousa-Uva M, Ahlsson A, et al. 2018 ESC/ EACTS guidelines on myocardial revascularization. Eur Heart J. 2019;40:87-165.

2. Montalescot G, Sechtem U, Achenbachm S, et al. ESC guidelines on the management of stable coronary artery disease. Eur Heart J. 2013;34:2949-3003.

3. Lloyd-Jones D, Adams RJ, Brown TM, et al. Heart disease and stroke statistics - 2010 update. Circulation. 2010;121(7):46-215.

4. Antman EM, Tanasijevic MJ, Thompson B, et al. Myocardial infarction redefined - a consensus document of the Joint European Society of Cardiology/American College of Cardiology committee for the redefinition of myocardial infarction: the Joint European Society of Cardiology/American College of Cardiology Committee. J Am Coll Cardiol. 2000;36(3):959-69.

5. Neumann FJ, Sousa-Uva M, Ahlsson A, et al. 2018 ESC/ EACTS Guidelines on myocardial revascularization. Eur Heart J. 2018;40(2):87-165.

6. Foy AJ, Dhruva SS, Peterson B, et al. Coronary computed tomography angiography vs functional stress testing for patients with suspected coronary artery disease. JAMA Intern Med. 2017;177(11):1623-31.

7. Shah BN, MacNab A, Lynch J, et al. Stress echocardiography in contemporary clinical cardiology: practical considerations and accreditation. Echo Research and Practice. 2018;5:1-6. and advantages and must be individualized according to the clinical condition of each patient. The development of new technologies and the refinement of the standard of existing ones, will lead to the progressive improvement of the images obtained by ultrasonography.

The digitization process, the use of images obtained by three-dimensional echocardiography in real time, the greater use of microbubble contrasts, the application of techniques derived from tissue Doppler are some examples of these resources that may be applied more frequently during the performance of the SE in the coming decades.

As it is a simpler method that does not require the use of radiation, which has been shown to induce cell damage, this study has focused on SE, which was necessary to diagnose $\mathrm{CAD}$ with sensitivities ranging from 44 to $100 \% .^{12-25}$

These data are extremely valid for actions to be taken in order to spread the method to the population, so that the diagnosis of CAD is made as early as possible and the appropriate treatment, so that we reduce the high incidence of deaths from cardiovascular causes in the country.

It is concluded, then, through this RSL and statistical tools that SE is an excellent tool for the diagnosis of CAD, and can be used more frequently in clinical practice.

8. Ministério da Saúde. Saúde Brasil 2017: Uma análise da situação de saúde e os desafios para o alcance dos objetivos de desenvolvimento sustentável. Brasília: DF, 2018.

9. Moher D, Liberati A, Tetzlaff J, et al. Preferred reporting items for systematic reviews and meta-analyses: the PRISMA statement. Ann Intern Med. 2009;151(4):264-69.

10. Wong WCW, Cheung CSK, Hart GJ. Development of a quality assessment tool for systematic reviews of observational studies (QATSO) of HIV prevalence in men having sex with men and associated risk behaviours. Emerg Themes Epidemiol. 2008;5:23.

11. Colorafi KJ, Evans B. Qualitative descriptive methods in health science research. HERD: Health Environments Research \& Design Journal. 2016;9(4):16-25.

12. Alfaro M, Palma G, Moreno O, et al. Experiencia con ecocardiograma de estrés dipiridamol y Reserva coronaria no invasiva: Comunicación preliminar. Rev Chil Cardiol. 2013;32(2):111-16.

13. Kamiya K, Sakakibara M, Asakawa N, et al. Cardiac magnetic resonance performs better in the detection of functionally significant coronary artery stenosis compared to single-photon emission computed tomography and dobutamine stress echocardiography. Circ J. 2014;78(10):2468-76.

14. Nagy Al, Sahlén A, Manouras A, et al. Combination of contrast-enhanced wall motion analysis and myocardial deformation imaging during dobutamine stress echocardiography. Eur Heart J Cardiovasc Imaging. 2015;16(1):88-95. 
Jorge João dos S. C. Filho, Paulo M. Neufeld • Stress echocardiography and coronary disease

15. Shehata M. Relation between ischaemic threshold measured during dobutamine stress echocardiography and coronary angiographic features. Kardiol Pol. 2014;72(12):1380-7.

16. Cusmà-Piccione $\mathrm{M}$, Zito $\mathrm{C}$, Oreto $\mathrm{L}$, et al. Longitudinal strain by automated function imaging detects single-vessel coronary artery disease in patients undergoing dipyridamole stress echocardiography. J Am Soc Echocardiogr. 2015;28(10):1214-21.

17. Aggeli C, Lagoudakou S, Felekos I, et al. Two-dimensional speckle tracking for the assessment of coronary artery disease during dobutamine stress echo: clinical tool or merely research method. Cardiovasc Ultrasound. 2015;24:13-43.

18. Utsunomiya $\mathrm{H}$, Hidaka $\mathrm{T}$, Masada $\mathrm{K}$, et al. Value of resting echocardiographic findings and dobutamine stress echocardiography for diagnosing myocardial ischemia in patients with suspected angina pectoris. Echocardiography. 2015;32(6):993-1002.

19. Park JH, Woo JS, Ju S, et al. Layer-specific analysis of dobutamine stress echocardiography for the evaluation of coronary artery disease. Medicine (Baltimore). 2016;95(32):4549.

20. Ejlersen JA, Poulsen SH, Mortensen J, et al. A comparison of the diagnostic value of 2D strain stress echocardiography, myocardial perfusion scintigraphy, and Duke treadmill score in patients suspected of coronary artery disease. Echocardiography. 2016;33(10):1523-31.

21. Rumbinaite E, Zaliaduonyte-Peksiene D, Lapinskas T, et al. Early and late diastolic strain rate vs global longitudinal strain at rest and during dobutamine stress for the assessment of significant coronary artery stenosis in patients with a moderate and high probability of coronary artery disease.
Echocardiography. 2016;33(10):1512-22.

22. Čelutkienè J, Burneikaitè G, Petkevičius L, et al. Combination of single quantitative parameters into multiparametric model for ischemia detection is not superior to visual assessment during dobutamine stress echocardiography. Cardiovasc Ultrasound. 2016;14(1):13.

23. Kataoka A, Scherrer-Crosbie M, Senior R, et al. Transient ischemic dilatation during stress echocardiography: an additional marker of significant myocardial ischemia. Echocardiography. 2016;33(8):1202-8.

24. Dattilo G, Imbalzano E, Lamari A, et al. Ischemic heart disease and early diagnosis. Study on the predictive value of $2 \mathrm{D}$ strain. Int J Cardiol. 2016 Jul 15;215:150-6.

25. Gurunathan S, Ahmed A, Vamvakidou A, et al. Diagnostic concordance and clinical outcomes in patients undergoing fractional flow reserve and stress echocardiography for the assessment of coronary stenosis of intermediate severity. J Am Soc Echocardiogr. 2018;31(2):180-86.

26. Bastos JLD, Duquia RP. Erratum para: Um dos delineamentos mais empregados em epidemiologia: Estudo transversal. Sci Med. 2013; 23:229-32.

27. Garg AX, Hackman D, Tonelli M. Systematic review and meta-analysis: when one study is just not enough. CJASN. 2008;3(1):253-60.

28. Wright RW, Brand RA, Dunn W, et al. How to write a systematic review. MD Clin Orthop Relat Res. 2007;455:23-9.

29. Pellikka PA, Nagueh SF, Elhendy AA, et al. American Society of Echocardiography recommendations for performance, interpretation, and application of stress echocardiography. J Am Soc Echocardiogr. 2007;20:1021-41. 